\title{
Use of Radio Equipment for Space Shuttle Navigation
}

\author{
EMIL R. SCHIESSER
}

\begin{abstract}
This paper discusses the role of radio navigation and communications equipment for Space Shuttle navigation. Radio navigation systems are discussed in terms of their ability to support Space Shuttle flight operations. A phased development of navigation capability from conventional trackers to relay satellites and navigation satellites is reviewed.
\end{abstract}

\subsection{INTRODUCTION}

QPACE Shuttle navigation is defined, in a narrow sense, as $D_{\text {the task of maintaining adequate knowledge of vehicle }}$ position and velocity. The state of the Orbiter in terms of this goal is described by a vector of at least six elements, three for position and three for velocity, at a given time. These are referred to as the "state vector" or simply the state.

Maintenance of the position and velocity at the user current time involves two processes: (1) the ability to determine the state vector by observation and (2) the propagation of such an initial vector forward without the benefit of such observations. These functions are referred to as state determination and state propagation.

Shuttle navigation will rely on a blend of ground-based and onboard systems. The onboard systems will be capable of state propagation at all times and will perform state determination during the latter part of the entry from orbit through landing and during the terminal phases of orbital rendezvous with another satellite. The ground-based system will be capable of accurate state propagation for free-flight phases during which the knowledge of contact forces (drag, thrust) is well known. State determination by the ground will be performed for all flight phases but is limited by the availability of observations on-orbit and in early descent. Trajectory designs must be heavily constrained in order to give the onboard and ground systems the opportunity to determine the state often enough for adequate state maintenance. The time interval over which adequate state maintenance can be performed using state propagation varies as a function of the uncertainty in the knowledge of forces acting on the vehicle that are modeled and/or measured.

Radio communication will be necessary in order for the ground and onboard capabilities to work as a coordinated system. Limited communications coverage for early (preTDRSS) Shuttle flights constrain trajectory design for those flights.

Radio navigation equipment will be used for state determination, while non-radio equipment will be used to support

Manuscript received J une 12, 1978; revised June 29, 1978.

The author is with the Lyndon B. Johnson Space Center, NASA, Houston, TX 77058 . state propagation. The use of radio equipment for state determination is discussed in this paper for the ascent, orbit, rendezvous, descent, and abort phases of Shuttle missions.

\subsection{ASCENT NAVIGATION}

\subsection{Onboard Ascent Navigation}

The onboard equipment planned for ascent navigation includes the master timing units, inertial measurement units (IMU's), computers, and crew displays and controls.

Prior to launch, the position of the navigation base on which the IMU's are placed will be loaded into the onboard computer in Earth-fixed coordinates. Velocity of the navigation base relative to the Earth will be set to zero. Any slight movement of the IMU's on the launch stand due to winds will be neglected. Position and velocity will be maintained in an inertial coordinate system starting (currently) $8 \mathrm{~s}$ prior to launch. The Earth-fixed position will be rotated to inertial coordinates to start the process. (Basic Shuttle coordinate systems are described in reference 1.) Onboard navigation formulation is presented in reference 2 .

State propagation will be accomplished by reading IMU accelerometer outputs to determine the amount by which the contact forces (push of Earth when on the pad and push of the engines and atmospheric drag, otherwise) have changed the velocity and by inclusion of the effects of gravitational force (central force, $\mu$, and Earth oblateness, J2) through modeling (ref. 3).

A single estimate of position and velocity will be obtained by using the middle value of each component of the sensed velocity from each of three IMU's (fig. 1). When only two are available, the average will be used. Use of the midvalue will protect the system from an IMU failure. The position and velocity carry a time tag. The time will be obtained by the computer about once each second, and in between it will be maintained using the computer oscillator at $25 \mathrm{~Hz}$. The sensed velocity data are tagged when obtained, and the position and velocity will receive that time tag when brought to that time.

The three-sigma $(3 \sigma)$ position and velocity errors at main engine cutoff (MECO) are expected to be about $4600 \mathrm{ft}$ and $20 \mathrm{ft} / \mathrm{s}$. The error in the velocity and position magnitudes (speed and radius) will be about $8 \mathrm{ft} / \mathrm{s}$ and $2000 \mathrm{ft}(3 \sigma)$. Use of the Department of Defense (DOD) global positioning satellite (GPS) system is being considered for the Orbiter since it will update the post-MECO state to accuracies of about $100 \mathrm{ft}$ and $0.6 \mathrm{ft} / \mathrm{s}$ in 2 to $4 \mathrm{~min}$. The GPS may not be useful before MECO because the external tank might block the line of sight between the Orbiter GPS antenna and most of the GPS satellites. 


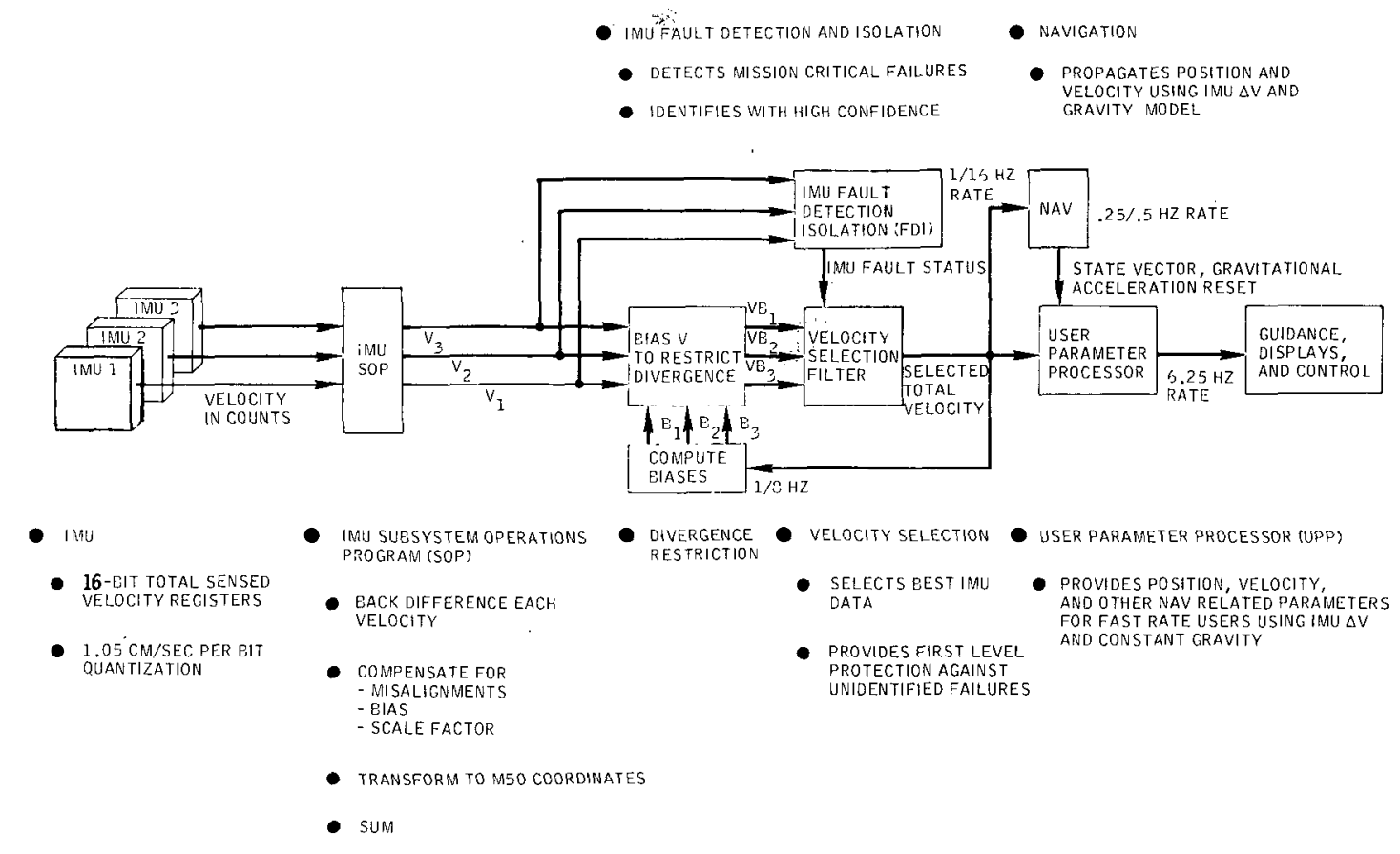

(BOTH NAV AND UPP BACK DIFFERENCE ON SELECTED TOTAL SENSED VELOCITY TO OETAIN IMU $\triangle$ V'S AT THEIR RESPECTIVE RATES.)

Figure 1. Onboard ascent navigation functional overview.

\subsection{Ground Ascent Navigation}

For early flights from Kennedy Space Center (KSC) the Mission Control Center (MCC) will monitor onboard navigation performance; update the onboard system, if necessary, to protect MECO conditions from gross onboard navigation errors; make abort decisions; monitor the health of the IMU's and other systems; and assist in redundancy management of onboard equipment.

In order to support these functions, the MCC will independently determine position and velocity through the use of $C$ and $S$-band tracking (fig. 2). The $C$-band trackers will provide time-tagged range, azimuth, and elevation measurements at a $10 / \mathrm{s}$ rate by skin tracking the Orbiter. The $S$-band transponder tracking will result in range, angles, and Doppler or rangechange measurements at a $10 / \mathrm{s}$ rate. Measurements from as many as one $S$-band and two $C$-band trackers will be processed together at 5 sets/s to provide parameters for display every $2 \mathrm{~s}$. Position will be readily determined. It will be difficult to determine velocity accurately using position-related observations prior to MECO-a region in which the forces acting on the Shuttle will not be well known to the ground processor.

The pre-MECO onboard state will be updated by adding corrections to it rather than by replacing it. This will be done to avoid saving a sliding window table of sensed velocity vectors onboard with which to propagate a received whole position/velocity vector to current time. The MCC will compare onboard state vectors received by telemetry with ground-based values, forming the differences required for uplink. The comparison of onboard velocity with ground-determined velocity from the tracking data filter will not be very useful because of the large uncertainty in the latter $(\sim 100 \mathrm{ft} / \mathrm{s})$. Instead, position differences at two different times will be used to compute the

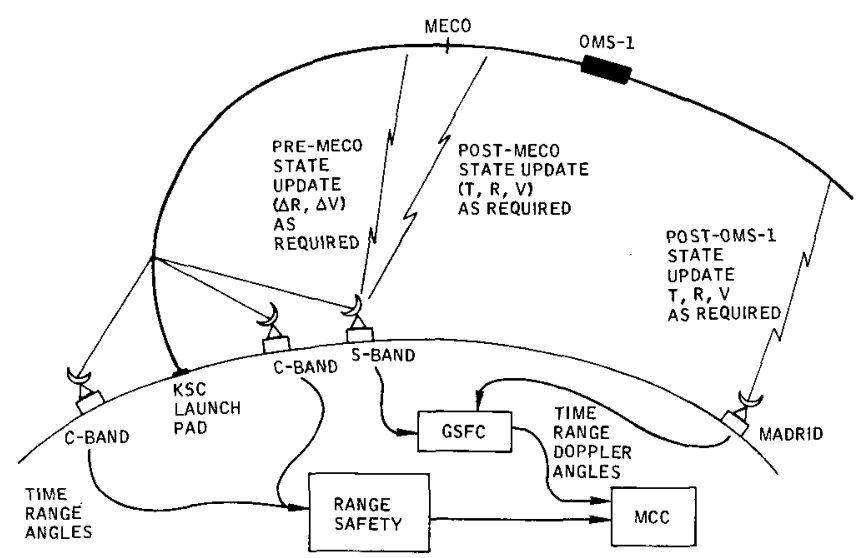

Figure 2. Ground ascent navigation.

velocity error, with the assumption that the position difference is constant if there is no velocity error. This will result in a longer equivalent data set being fit and is possible because of the indirect use of the IMU-sensed velocity data on the ground through the telemetered onboard position information. Direct use of telemetered-sensed velocity data could be used to achieve even better performance; however, there is a desire to keep the tracking data processing filter independent of the onboard systems.

After MECO, $1 \mathrm{~min}$ of free flight will be allowed for the ground to compute position and velocity for abort decisions and a possible Orbiter state vector update for the first orbital flight test. During free flight (following MECO), the forces acting on the Orbiter will be much better known to the MCC than for powered flight. This will result in improved $\mathrm{MCC}$ velocity determination-about $30 \mathrm{ft} / \mathrm{s}(3 \sigma)$. Speed will be determined 
to about $8 \mathrm{ft} / \mathrm{s}(3 \sigma)$. Should a state vector update be required, the onboard software will receive and bring to current time an uplinked whole state vector since this can be done without the use of IMU-sensed velocity. The post-MECO tracking will rely on the Bermuda $S$-band system for the first flight since it may be difficult for the $C$-band tracker to distinguish between the large fuel tank and the Orbiter, which separate at a relative speed of about $4 \mathrm{ft} / \mathrm{s}$ just after MECO. The $C$-band systems have better angle measurements, and the angle errors are a major contributor to post-MECO ground-determined state vector error. Speed accuracy requirements post-MECO are quite tight-in the 4- to $10-\mathrm{ft} / \mathrm{s}$ range, being driven by abort decisions and the potential use of the vector for an abort into a oncearound trajectory.

There will be two orbit-shaping maneuvers: one 2 min after MECO and one about half of a revolution later. These are called OMS-1 and OMS-2 after the orbital maneuvering system (OMS), which is used to execute the burns. After OMS-2, the Orbiter will nominally be in a 150-nmi circular orbit (ref. 4). There will be provision for a post-OMS-1 state vector update over Madrid for the first orbital flight test. The vector would be based on about 1 min of tracking data. This state would be predicted forward through a simulated OMS-1 maneuver before being sent up.

The Tracking and Data Relay Satellite system (TDRSS), which will be available by 1981 , will not be useful for launch navigation since only the component of the velocity vector which lies along the Orbiter-to-TDRS line of sight is measured at any one time, compared to all three position components for $C$-band tracking and four state vector components for $S$ band tracking. Use of the TDRSS for launch $S$-band communications will result in loss of ground $S$-band tracking support since only one $S$-band system can communicate with the Orbiter at a time.

\subsection{ORBITAL NAVIGATION}

The ground will provide initial state vectors to the onboard system as required for onboard propagation.

\subsection{Onboard Orbital Navigation}

The Orbiter will maintain current position and velocity through periodic propagation using numerical integration of acceleration based on theoretical force models. Orbital maneuvers are an exception, in which case sensed velocity and a gravitational force model are used. In addition to attitude-dependent drag (based on IMU gimbal angles and a Jacchia atmospheric density model (ref. 5)), a simple workhorse gravity model will be used onboard, which includes the central force, zonal terms through fourth order, and second-order tesseral terms (ref. 6).

\subsection{Ground Orbital Navigation}

\subsubsection{Pre-TDRSS}

For the OFT and early operations orbital phase, the MCC will obtain $C$ - and $S$-band tracking observations. A curve based on numerical integration of acceleration from force models and an initial estimate of the state will be fit to the tracking data. Adjustments will be made to the initial estimate until a

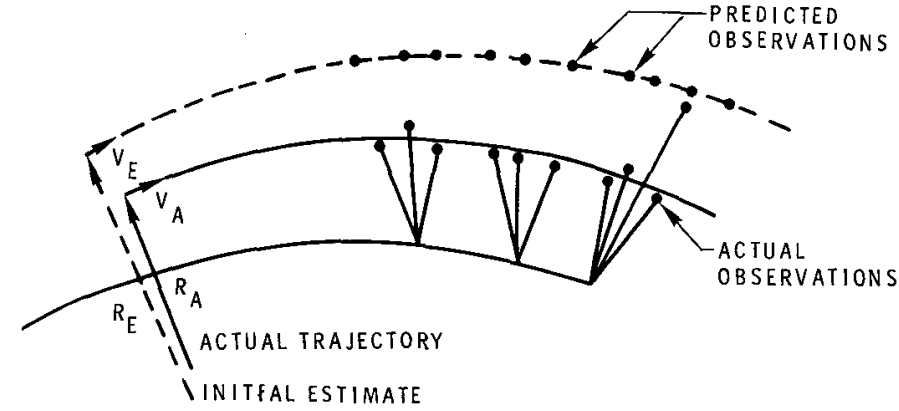

Figure 3. Orbital navigation technique.
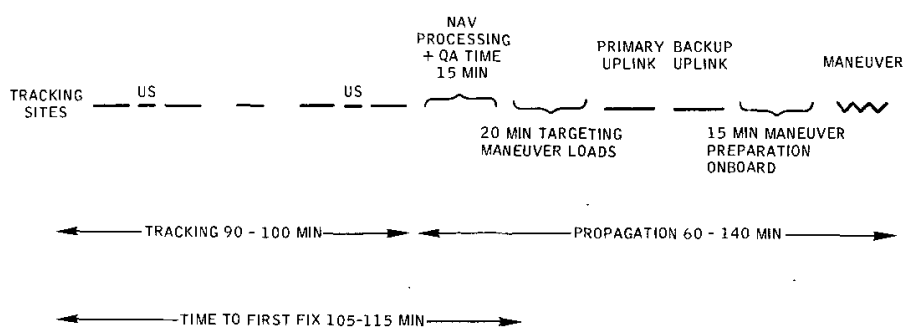

Figure 4. MCC orbital navigation time line-direct tracking/ communications.

good fit is achieved (fig. 3). The resulting position and velocity estimate will be sent to the Orbiter by command uplink. The MCC will also compute maneuver characteristics that will be sent to the Orbiter for onboard guidance to execute. MCC ground $C$ - and $S$-band navigation formulation are described in references 7 and 8 .

The tracking time will be determined based on the need to observe spacecraft motion free from maneuvers for about a revolution in order to determine the orbital period for downtrack position prediction. Sparse direct tracking coverage can result in the need to extend the interval so that sufficient data will be available.

For essential maneuvers, there must be protection from potential tracker failure, so that two trackers on each end of the tracking interval are required. The time between vector availability and the maneuver is controlled by maneuver preparation activities: time required for maneuver targeting and the requirement for a primary and a backup uplink site. Figure 4 shows that 150 to $240 \mathrm{~min}$ or more are required from the start of tracking to the initiation of the maneuver. Two planned orbital maneuvers can be placed no closer than the groundtracking, processing time, and uplink availability allow unless the maneuvers can be targeted and executed as a pair. This is sometimes possible when the second maneuver is not sensitive to the error in performing the first.

State propagation, especially for the downtrack position component, is very susceptible to small translational perturbations due to vents, drag, and attitude maneuvers with thrusters that are not perfectly balanced so that they produce not only torque but also a net translational acceleration.

Typical $3 \sigma$-downtrack position accuracy, after predicting a revolution into the future for Skylab, was $1500 \mathrm{ft}$. Skylab was 
free of trajectory perturbations and it was possible, with some care, to achieve $9000-\mathrm{ft}, 3 \sigma$ position accuracies after 1 day. During the Apollo-Soyuz Test Project (ASTP), the command/ service module often used uncoupled jets for attitude control. During such times, position when predicted one revolution ahead was accurate to about $60,000 \mathrm{ft}(3 \sigma)$. Preliminary projections for Shuttle place it between Skylab and ASTP, with expected $3 \sigma$ one-revolution prediction accuracy in the 9000 to 30,000 -ft range, depending on the attitude control and vent activity.

\subsubsection{Post-TDRSS}

The availability of the TDRSS will remove the need for direct uplink sites and provide tracking data independent of the groundtrack. A description of MCC TDRSS ground navigation formulation is contained in references 9 and 10 .

The TDRSS will provide two-way range change (Doppler) over a path from the master station on the ground, through the TDRSS, to the Orbiter transponder, and back. Nominally, two relay satellites separated by a large Earth central angle will be seen within a single revolution of the Orbiter about the Earth. The use of Doppler data through both satellites over one Orbiter revolution should result in adequate state vectors.

Should only a single relay satellite be available, then two passes are desired. This would require about $135 \mathrm{~min}$. One pass of 50 or so min followed by another half pass would be suitable as a minimum tracking interval (115 $\mathrm{min})$.

A rough time line (fig. 5) shows that 120 to $195 \mathrm{~min}$ will be required from the start of tracking to maneuver execution.

Availability of the GPS for navigation and TDRSS for communications will greatly reduce, if not remove, navigation restrictions on flight planning, trajectory design, and flight control (fig. 6). Adequate orbital navigation could be performed using the phase-1 six-satellite GPS constellation available in 1979 but would constrain mission time lines because up to 50 min would be required to obtain the state. Determination of Orbiter state on-orbit, using the 18- to 24-satellite constellation expected in the mid-1980's, will be a relatively easy task since four satellites would always be visible. The state could be determined within a couple of minutes to about $100 \mathrm{ft}$ in position and $0.6 \mathrm{ft} / \mathrm{s}$ in velocity $(3 \sigma)$. This could be done continuously at about a 4 -s rate.

\subsection{RENDEZVOUS NAVIGATION}

The early maneuvers in a rendezvous sequence will be targeted by the ground and must be planned around groundtracking coverage. The terminal phase maneuvers will be determined onboard and must be planned in accordance with onboard relative observation (spacecraft to spacecraft) restrictions.

\subsection{Onboard Rendezvous Navigation}

The terminal rendezvous phase navigation will be performed with the use of the star tracker and/or $K u$-band rendezvous radar, which will provide relative observations between the Orbiter and the target spacecraft.

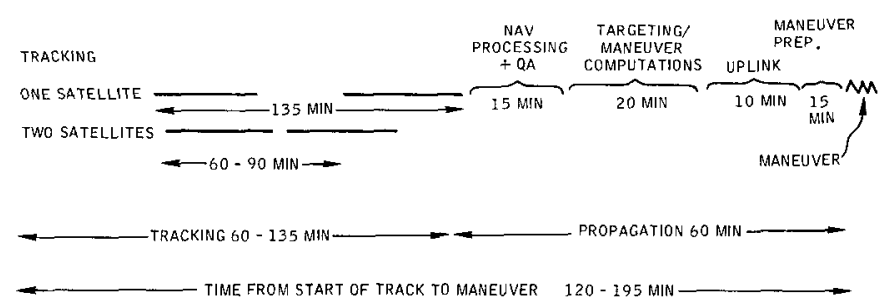

Figure 5. MCC orbital navigation time line for TDRSS tracking/ communication.

\section{DIRECT TRACKING \\ C-BAND \\ S-BAND \\ RELAYED TRACKING
TDRSS \\ GPS UUNDER CONSIDERATIONI \\ NUMBER GPS
SATELLITES \\ IUS/RENDEZVOUS OBJECTS \\ DIRECT TRACKING \\ C-BANO \\ S-BAND - NASA \\ - DOD \\ RELAYED TRACKING}

TDRSS

Figure 6. Orbital navigation system schedule.

The star tracker will provide angles out to a range of about $300 \mathrm{nmi}$. The rendezvous radar will have a $10 \mathrm{nmi}$ skin track capability for a $1-\mathrm{m}^{2}$ target, providing range, angles, angle rate, and range rate data.

The star tracker measures two components of the relative position at any one time, so it is a relatively weak navigation device. By contrast, the rendezvous radar measures all components of relative position and velocity. The star tracker poses numerous other operational difficulties as well. These include recognition of the proper object to track, limitations on target brightness, lighting requirements on the target, and proximity of Sun line of sight with respect to instrument field of view.

The rendezvous radar will be limited primarily by the short range over which it can track passive objects.

\subsection{Ground Rendezvous Navigation}

There are some constraints associated with rendezvous. Time lines must be worked until proper tracking and communication coverage are available for the early maneuvers targeted by the ground. Use of the GPS for navigation would remove these constraints assuming that the satellite to be met is not maneuvering and its state can be accurately determined by the ground and propagated accurately onboard. Availability of TDRSS for communications and tracking will be a big improvement, especially for flights in orbits inclined more than about 50 degrees. 


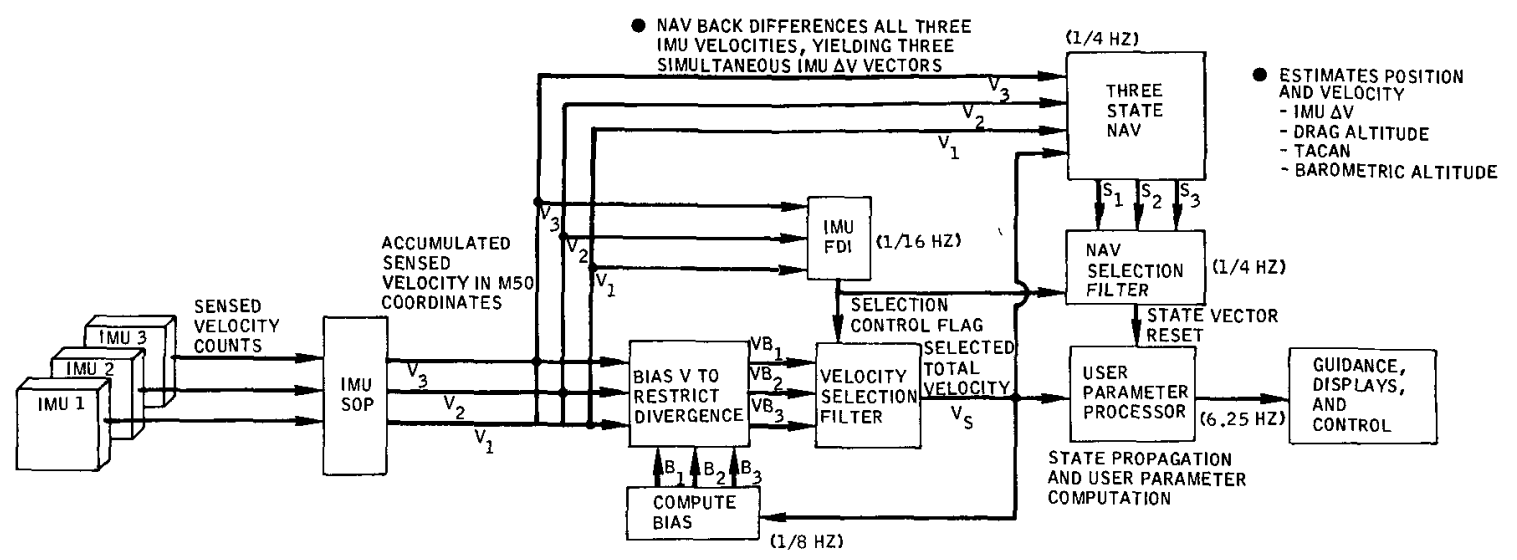

Figure 7. Onboard entry triple state navigation functional overview.

\subsection{ENTRY NAVIGATION}

The MCC will provide initial position and velocity to the Orbiter for entry. For OFT-1, about $2 \mathrm{~h}$ of state propagation time lie between the last data processed and the deorbit maneuver. Errors on knowledge of actual position at the time of deorbit are expected to be on the order of $30,000 \mathrm{ft}(3 \sigma)$ based on rough estimates of vent and attitude-control thrusting effects (ref. 11).

\subsection{Onboard Entry Navigation}

The onboard navigation system will maintain position and velocity with the use of IMU-sensed velocity after contact acceleration exceeds $1000 \mu \mathrm{g}$. Prior to that, state propagation will be accomplished by using attitude-dependent drag and gravitational force models (ref. 12).

Knowledge of altitude is expected to deteriorate rapidly with time (ref. 13). Left unattended, the altitude error at the end of the communications blackout at about $170,000 \mathrm{ft}$ altitude could be about $60,000 \mathrm{ft}$. The error in altitude will be reduced by the use of "drag altitude updating." The drag acceleration measured by the IMU is a function of vehicle shape, speed, and atmospheric density. The density in turn varies with altitude. Altitude is computed deterministically, given the other parameters, and presented to the entry navigation data filter to correct radius and, to a limited extent, other components of position and velocity. This results in altitude errors after blackout of about $12,000 \mathrm{ft}(3 \sigma)$.

Three position and velocity vectors, one for each IMU, are maintained in each of four primary computers (fig. 7). These are initialized from one vector about $2 \mathrm{~h}$ before deorbit and carried down to microwave scanning beam landing system (MSBLS) acquisition. If two IMU's fail, navigation is degraded, but recovers if there is an opportunity to select the one remaining good state vector.

The management of the three redundant IMU's will use builtin tests, communication fault tests between the IMU and computer, and software that takes advantage of a purposely skewed orientation of the output axes of the accelerometers and gyros between any pair of the three platforms.

At about $160,000 \mathrm{ft}$ altitude (fig. 8), the first tactical air navigation (TACAN) onboard range and bearing data should

\begin{tabular}{|l||lll|}
\hline $\begin{array}{l}\text { APPROXIMATE } \\
\text { ALTITUDE (KFT) }\end{array}$ & 280 & 260 & 150 \\
\hline TACAN & & & \\
\hline DRAG ALTITUDE & & & \\
\hline $\begin{array}{l}\text { BAROMETRIC } \\
\text { ALTIMETER }\end{array}$ & & & \\
\hline MSBLS & & \\
\hline
\end{tabular}

\section{ITS ACQUIRED}

ZZD ACQUIRED AND USED

Figure 8. Altitudes for planned use of entry navigation data.

be acquired. The first TACAN data to be processed must be valid because the navigation data filter will assume they are valid and adjust the poorly known state vector accordingly. Erroneous observations will be edited via a residual test if the state vector is well known and its associated error covariance matrix is small.

The approach and landing tests (ALT) performed in 1977 demonstrated that TACAN data are subject to a variety of errors, including 40-degree biases in bearing, which result from a misunderstanding in the receiver about which lobe of the ground transmitter it is to lock onto. The device is not very inclined to indicate that data are invalid during the acquisition process. Above about 45 degrees elevation of the Orbiter to the ground transmitter, the bearing data drop out, but the range is still valid. TACAN experience with the type of units on the Orbiter at high relative velocities encountered after blackout (6000 to $7000 \mathrm{kn}$ ) is nonexistent (not experienced on ALT or aircraft).

For the first orbital flight tests, the TACAN data will initially be inhibited until the crew and/or the ground have determined that good data are available. The TACAN data quality checks include requirements for two of the three units onboard to agree before any data are sent to the filter for use in updating position and velocity. Further, after acquisition the first few observations are discarded to avoid bad data. It has been observed that two or three independent onboard TACAN receivers can have a series of errors in the bearing measurements of the same size, escaping direct comparison tests. The MCC will use $C$-band data to compute Orbiter position and from 
that predict TACAN observations for comparison with onboard observed values. This can be done well since the MCC position vectors should be good to about $1500 \mathrm{ft}(3 \sigma)$.

The air data system will provide barometric altitude. These data will be inaccurate near Mach 1 , and because necessary calibration data are not available, barometric data are not planned for use above Mach 0.75 (about 33,000 ft) for the first flight unless insufficient TACAN data are available for altitude determination.

At about $20,000 \mathrm{ft}$ altitude, the MSBLS will be acquired. The MSBLS is comprised of two ground units/antennas, one for emitting a wedge elevation angle beam and the other for range and bearing. Measurements are obtained onboard through three interrogating/receiving units. Landing navigation is performed easily using the MSBLS because the measurements provide fresh information on all components of position each processing cycle, and the measurement errors are small enough to extract adequate velocity information when used with IMUsensed velocity. Radar altimeter observations can be made up to $5000 \mathrm{ft}$ altitude; but because of the availability of MSBLS and because of terrain variation effects, the radar altimeter will be used only below $500 \mathrm{ft}$ altitude. The radar altimeter measurements are used to replace the altitude component of the state for user-parameter computations and are not presented to the slower running navigation filter. The crew can manually land the Orbiter from $20,000 \mathrm{ft}$ altitude down (as demonstrated on ALT) without the benefit of the autoland navigation capability.

\subsection{Ground Entry Navigation}

For early OFT flights the DOD Western Test Range and Edwards Air Force Base $C$-band trackers will be used to determine position and velocity (fig. 9). The same computer programs are used in the MCC for launch and entry, including the Kalman filter for state determination and the delta state update technique (ref. 14).

In addition to TACAN data management support, the MCC will be capable of updating onboard knowledge of position after blackout at an altitude of about $150,000 \mathrm{ft}$. Orbiter downtrack position maneuverability decreases rapidly following blackout so that it is important that the onboard knowledge of downrange position is updated in the 130,000 - to $150,000-\mathrm{ft}$ altitude region or higher. It will be relatively easy for the ground to provide such an update since the Orbiter position will be easy to determine using $C$-band tracking. Velocity will not likely be updated because the quality of ground-determined velocity is expected to be poor.

$C$-band skin track data will be available 1 to $2 \mathrm{~min}$ before the ground and Orbiter will be able to communicate via $S$-band. This will enable the MCC to be prepared at $S$-band blackout exit with ground control instructions as required. An update to the state vector in the form of a position change in runway coordinates will require onboard telemetered position vectors via $S$-band through the Edwards/Buckhorn communication equipment.

Studies indicate that downtrack navigation errors as large as $10 \mathrm{nmi}$ can be handled by entry guidance, which must satisfy contact acceleration, ranging to the landing site, and other re-

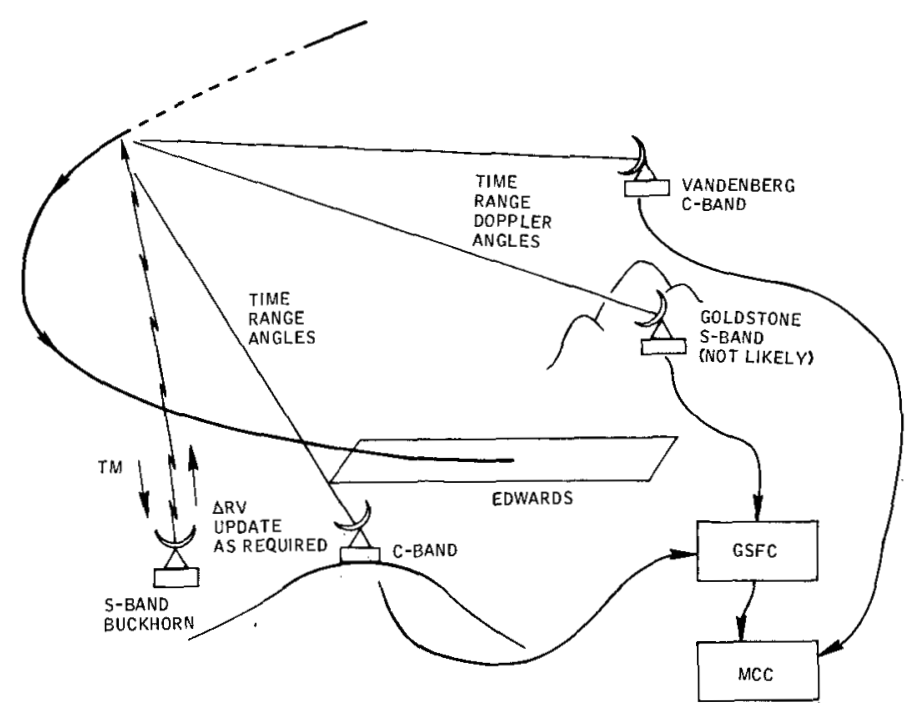

Figure 9. OFT MCC entry navigation.

quirements. The actual vehicle position can deviate from the nominal at deorbit by 100 to $200 \mathrm{nmi}$ without seriously affecting entry.

In the mid 1980's, GPS may be used to provide accurate state updates between deorbit and entry blackout. This is not possible with direct or TDRSS tracking. During radio blackout, the Orbiter must propagate the state using the IMU even with GPS, although blackout may be shorter for back-looking transmission than for forward looking because of the orientation of the ion sheath. The GPS would provide accurate post-blackout navigation capability ( $200 \mathrm{ft}$ and $1.5 \mathrm{ft} / \mathrm{s})$, eliminating the need to use TACAN and barometric altimeter data. The TACAN receivers could be removed, given sufficient redundancy in the number of GPS receiver/processor units. This could take place after the 24-satellite constellation is operational such that sufficient GPS satellites are always visible to the Orbiter.

\subsection{ABORTS}

There are a number of abort trajectories that require accurate navigation (ref. 15). These include the abort once-around during which the Orbiter returns to a landing site after going once around the Earth instead of into a stable orbit; the returnto-launch site (RTLS) in which the Orbiter separates from the external tank and does extensive maneuvering to return to the launch site runway; the abort to orbit (in the event of an early MECO, it is possible to fire the OMS to achieve a lower (105 nmi) circular orbit than nominal). In addition, there is the contingency deorbit into non-nominal landing areas. Onboard navigation support of the RTLS is adequate. State maintenance with the IMU's benefits from small errors that result from the short operating time on the IMU's and the good prelaunch alignment. In addition, TACAN and barometric altimeter data are available, followed by MSBLS. The abort to orbit is similar to nominal navigation, although the lower orbit results in greater drag forces and correspondingly larger uncertainties as well as less direct tracking coverage. The abort once-around, because of the sparse communications and tracking, might result in very poor post-blackout entry conditions except for the crosstrack steering required to get to a runway to the side of the orbital 
groundtrack. This maneuvering appears to place a large 15 to $20 \mathrm{nmi}$ downtrack error in a crosstrack direction, which makes it much easier to maneuver out.

A contingency deorbit into non-nominal landing sites is difficult to support. Groundtracking and TACAN support down range of the landing site will often be lacking; and, on OFT-1, the barometric altimeter is expected to be inaccurate above Mach 0.75 .

Availability of TDRSS will alleviate some of the stress on the orbital navigation and communications. The GPS would provide significant relief in abort situations, especially for descent into contingency landing sites and for the abort once around. The ground is capable of supporting an RTLS using direct $C$ - and $S$-band tracking.

\subsection{CONCLUSIONS}

Shuttle navigation and communication requirements place some constraints on Orbiter trajectory design and operations.

The TDRSS will remove most communication constraints but will do little to remove Shuttle navigation trajectory and time-line constraints because of the relatively long time required for orbit state determination and inability to support launch and entry navigation. Use of GPS in the 1984/85 time frame would provide time-line relief and would reduce any uncertainties that may remain after current navigation systems have matured.

\section{ACKNOWLEDGMENT}

Information was obtained primarily through past discussions with G. deVezin (rendezvous navigation), P. Pixley (ground ascent/descent navigation), R. Savely (onboard navigation), W. Wollenhaupt (ground orbital navigation), T. Sheehan and B. Batson (navigation and communication hardware characteristics).

Special effort was made by P. Marsden, S. Sanford, K. Tu, and M.S. Burton in the preparation/editing of the text and figures by working around their busy schedules.

\section{REFERENCES}

1. Davis, Larry D.: Coordinate Systems for the Space Shuttle Program. NASA TM X-58153, 1974.

2. Space Shuttle Orbital Flight Test-Level C Functional Subsystem Software Requirements Document. Guidance, Navigation, and Control, Part B, Navigation Ascent/RTLS, SD 76-SH-0005B, Rockwell International, Sept. 1977.

3. Cockrell, B. F.; and Williamson, Bruce: Space Shuttle Astrodynamical Constants. JSC IN 78-FM-32, June 1978.
4. Flight Analysis Branch: OFT-1 Operational Flight Profile-Volume III, Ascent. JSC 77-FM-59, April 1978.

5. Jacchia, L. G.: New Static Models of the Thermosphere and Exosphere With Empherical Temperature Profiles. Smithsonian Astrophysical Observatory, 1970.

6. Space Shuttle Orbital Flight Test-Level C Functional Subsystem Software Requirements Document. Guidance, Navigation, and Control, Part B, Navigation Onorbit, SD 76-SH-0006A, Rockwell International, July 1977.

7. Mathematical Physics Branch: RTCC Requirements for Apollo 14: Trajectory Prediction Program Description. JSC IN 70-FM-200, Dec. 24, 1970.

8. Schiesser, Emil R.; deVezin, Howard G.; Savely, Robert T.; and Oles, Michael J.: Basic Equations and Logic for the Real-Time Ground Navigation Program for the Apollo Lunar Landing Mission. JSC IN 68-FM-100, April 15, 1968.

9. Osburn, R. K.; and Wollenhaupt, W. R.: OPS MCC Ground Navigation Program Level C Display and Control Requirements (to be published).

10. Williamson, J. B.: OPS MCC Ground Navigation Program. Level C Software Requirements Orbit Determination Processing Formulations Document. JSC 78-FM-30 (to be published).

11. Heath, David; Carman, Gilbert; Payne, Dan; Gonzales, Lazarus and Montez, Moises, Flight Analysis Branch; Hiott, James M.; Ruda, Roger; Hochstein, Allan; Simpson, Don; Bump, Paul; and Kyle, Howard C., McDonnell Douglas Technical Services Co.: OFT-1 Operational Flight Profile-Volume V, Deorbit Through Landing. JSC IN 77-FM-59, April 1978.

12. Space Shuttle Orbital Flight Test-Level C Functional Subsystem Software Requirements Document. Guidance, Navigation, and Control. Part B, Entry Through Landing Navigation, SD 76-SH$0004 \mathrm{C}$, Rockwell International, Oct. 1977.

13. Montez, Moises: OFT-1 Reference Flight Profile-Deorbit Through Landing, Appendix B-Monte Carlo Dispersion Analysis. JSC IN 77-FM-36, Feb. 1978.

14. Mathematical Physics Branch, JSC; Mathematical Physics Support Group, McDonnell Douglas Technical Services Co.; and Bill Lear, TRW Systems: Requirements for Shuttle OFT High-Speed Trajectory Determination (Launch and Landing Phases). JSC IN 76FM-86, Oct. 4, 1976

15. Flight Analysis Branch: OFT-1 Operational Flight Profile-Volume VI, Abort Analysis. JSC IN 77-FM-59, May 1978.

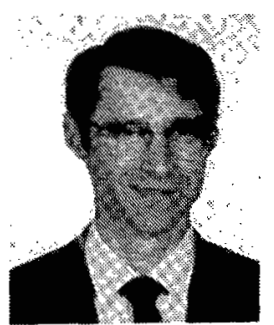

Emil R. Schiesser was born in Ladysmith, Wisconsin, in 1937. He received a B.S. in physics from Northern Illinois University in 1959 and an M.S. in 1961.

Mr. Schiesser joined the Space Task Group at Langley Research Center in 1961 and moved with that group to Houston with the formation of the Manned Spacecraft Center in 1962. He performed navigation accuracy assessment studies during projects Mercury and Gemini and directed the development of Apollo ground navigation software formulation. He has been Chief of the Mathematical Physics Branch (MPB) since 1973. MPB was responsible for the Apollo Soyuz Test Program (ASTP) and Skylab ground and onboard operational navigation and is responsible for shuttle ground and onboard navigation software formulation, flight techniques support, and real time navigation console operations. 